\title{
Recherches sur l'oncomiracidium des Capsalidae (Monogenea)
}

\author{
par A. LAMBERT \\ Laboratoire de Parasitologie comparée \\ Université des Sciences et Techniques du Languedoc, F 34060 Montpellier Cedex
}

\section{Résumée.}

Nous avons étudié en détail les cellules ciliées et la chétotaxie de la larve de Benedenia monticelli (Parona y Perugia, 1895), parasite de Mugil cephalus L.

Nos observations sur l'oncomiracidium de Trochopus pini (Van Beneden et Hesse, 1863) et Entobdella soleae (Van Beneden et Hesse, 1863) prouvent qu'il y a un plan commun d'organisation de ces structures chez ces Capsalidae.

Ce plan est différent de celui mis en évidenze chez les Dactylogyroïdea d'une part et les Polyopisthocotylea d'autre part.

Le type chétotaxique Capsaloïdea est original et représente, peut-être un schéma ontogéniquement plus avancé comme le sont chez ces Monogènes l'armature du hapteur et la disposition du système excréteur.

\section{Summary.}

Investigations on the oncomiracidium of Capsalidae (Monogenea).

A detailed study of the ciliated cells and chetotaxy of the larva of Benedenia monticelli (Parona y Perugia, 1895) parasite of Mugil cephalus L. is carried out.

Observations on the oncomiracidium of Trochopus pini (Van Beneden and Hesse, 1863) and Entobdella soleae (Van Beneden and Hesse, 1863) reveal a common plan of these structures among those Capsalidae.

The pattern is different from that observed in the Dactylogyroïdea on one hand and Polyopisthocotylea on the other hand.

The chetotaxic type of Capsaloïdea is original and it could, perhaps, be more advenced ontogenetic scheme as are the haptoral sclerites and the excretory system among these Mono. genea.

Accepté le 3 février 1978. 
Dans des travaux précédents (Lambert 1976-1977 $a$-1977 b), nous avons démontré l'importance de la chétotaxie de l'oncomiracidium des Monogènes pour juger des affinités phylétiques des grands groupes et ainsi aborder sur une base originale le problème de leur phylogénèse.

Chez les Monopisthocotylea, nous connaissons jusqu'à présent des espèces et des genres de Dactylogyroïdea et Euzetrema knoepffleri; de plus, nous avons montré (Lambert, 1978) que Tetraonchus monenteron (Tetraonchoïdea) présentait de grandes affinités avec les Dactylogyroïdea.

Nous décrivons pour la première fois cette disposition anatomique chez trois Monogènes (Benedenia monticelli, Entobdella soleae et Trochopus pini) appartenant à un autre groupe important des Monopisthocotylea: les Capsalidae.

\section{I. - Benedenia monticelli}

\section{A. - Cellules ciliées (fig. 1 et 2).}

Les cellules ciliées épidermiques sont disposées en quatre zones nettement séparées: une zone céphalique antérieure, deux zones pleurales et une zone haptoriale postérieure.

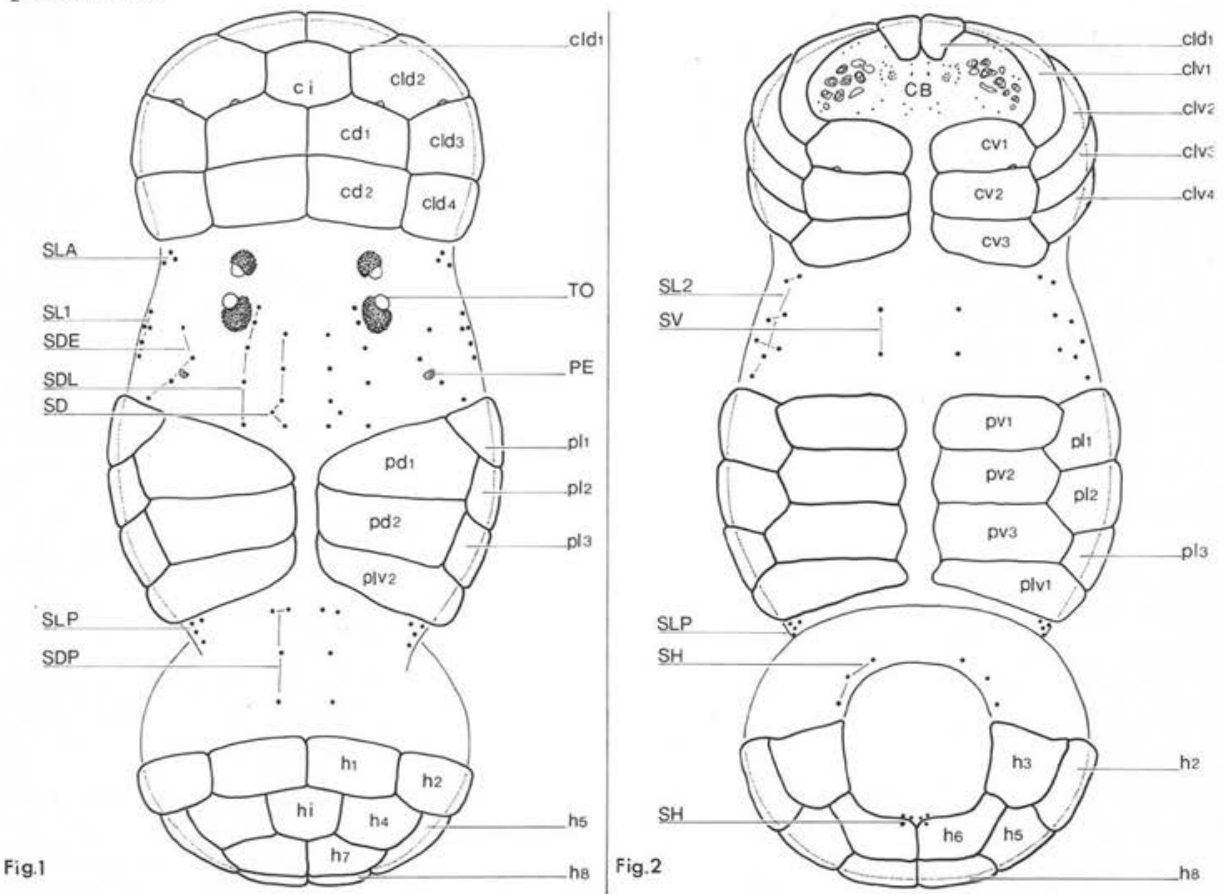

Fig. 1 et 2. Benedenia monticelli (Parona et Perugia, 1895). Représentation schématique des cellules ciliées et de la chétotaxie de l'oncomiracidium.

Fig. 1: face dorsale; fig. 2: face ventrale.

Nomenclature des cellules ciliées et des sensilles: voir texte.

CB : Cadre buccal ; PE : Pore excréteur; TO : Taches oculaires. 
- La zone céphalique comprend 27 cellules jointives qui coiffent la partie apicale de la larve et entourent ventralement le cadre buccal.

- Sur la face dorsale nous avons compté: une cellule médiane impaire (CI) entourée par 6 paires de cellules symétriques dont 2 sont dorsales $\left(C_{1}\right.$ et $\left.C_{2}\right)$ et 4 latéro-dorsales $\left(\mathrm{CLD}_{1}\right.$ à $\left.\mathrm{CLD}_{4}\right)$. Les 2 cellules latéro-dorsales les plus antérieures $\left(C L D_{1}\right)$ se prolongent légèrement sur la face ventrale et font saillie frontalement en avant du cadre buccal.

- Sur la face ventrale il existe 7 paires de cellules dont 3 sont ventrales $\left(\mathrm{CV}_{1}\right.$ à $\left.C_{3}\right)$ et 4 latéro-ventrales $\left(C_{1} V_{1}\right.$ à $\left.C L V_{4}\right)$ symétriques. Les 3 paires de cellules ventrales s'affrontent sans se toucher de part et d'autre du plan médio-sagittal.

Nous avons observé entre les cellules $C_{1}$ et $\mathrm{CLD}_{2}, \mathrm{CLD}_{3}$ et $\mathrm{CLD}_{2}, \mathrm{CV}_{1}$ et $\mathrm{CV}_{2}$ une toute petite zone où les limites des cellules ciliées sont séparées; nous interprétons ces formations comme étant peut-être le débouché de glandes céphaliques.

- Chaque zone pleurale comprend 10 cellules jointives: 2 cellules dorsales $\left(\mathrm{PD}_{1}\right.$ et $\left.\mathrm{PD}_{2}\right), 3$ cellules ventrales $\left(\mathrm{PV}_{1}\right.$ à $\left.\mathrm{PV}_{3}\right)$ et 3 cellules latérales $\left(\mathrm{PL}_{1}\right.$ à $\left.\mathrm{PL}_{3}\right)$ reliant les cellules ventrales et dorsales, et, postérieurement, 2 cellules, l'une latéroventrale $\left(\mathrm{PLV}_{1}\right)$, l'autre latéro-dorsale $\left(\mathrm{PLV}_{2}\right)$. Ventralement et dorsalement ces cellules s'affrontent sans contact, de part et d'autre du plan de symétrie. Les 2 zones pleurales forment ainsi une sorte de manchon autour de la région médiane de la larve, uniquement interrompu par une étroite bande non ciliée dorsale et ventrale.

- La zone haptoriale comporte 17 cellules jointives qui couvrent dorsalement la moitié postérieure du hapteur. D'avant en arrière il y a par hémicorps : 3 cellules $\left(\mathrm{H}_{1}\right.$ à $\left.\mathrm{H}_{3}\right)$; puis, 3 cellules $\left(\mathrm{H}_{4}\right.$ à $\left.\mathrm{H}_{6}\right)$ de part et d'autre d'une cellule impaire $(\mathrm{HI})$ et enfin 2 cellules postérieures $\left(\mathrm{H}_{7}\right.$ et $\left.\mathrm{H}_{8}\right)$.

B. - Chétotaxie (fig. 1 et 2).

Les sensilles étant disposées symétriquement, nous donnons, pour chaque groupe, leur nombre pour un hémicorps.

Face dorsale, on distingue :

- 5 sensilles alignées dorsalement (SD) dont les 3 postérieures sont plus rapprochées. Ces sensilles s'alignent près du plan médio-longitudinal dans une zone comprise entre les taches oculaires postérieures et les premières cellules ciliées des zones pleurales $\left(\mathrm{PD}_{1}\right)$.

- 5 sensilles dorsales plus latérales (SDL). Elles sont plus espacées que les précédentes; les 2 plus antérieures étant situées à la limite du bord axial des taches oculaires postérieures.

- 4 sensilles entre le groupe dorso-latéral et le bord du corps, au niveau du pore excréteur (SDE). Deux sensilles sont antérieures par rapport à cet orifice, les 2 autres postérieures. Les 3 plus postérieures sont alignées obliquement.

- 4 sensilles dorsales postérieures (SDP) entre les zones ciliées pleurales et haptoriales. Les 2 sensilles antérieures forment un doublet rapproché, les 2 autres sont espacées régulièrement. 
Face ventrale :

Il y a seulement 2 sensilles ventrales (SV) qui forment avec leurs symétriques un carré situé entre les cellules ciliées céphaliques et pleurales.

Latéralement, on distingue :

- 3 sensilles latérales antérieures (SLA) juste en arrière des cellules ciliées céphaliques au niveau des $\mathrm{CLD}_{4}$.

- 5 sensilles latérales $\left(\mathrm{SL}_{1}\right)$ situées entre le groupe latéral antérieur et les cellules ciliées pleurales $\left(\mathrm{PL}_{1}\right)$. Deux d'entre elles forment un doublet transversal.

- 6 à 8 sensilles latérales plus ventrales $\left(\mathrm{SL}_{2}\right)$ alignées irrégulièrement sur tout l'espace compris entre les cellules ciliées céphaliques et pleurales.

- 8 à 12 sensilles latérales postérieures (SLP) comprises entre les cellules ciliées pleurales et le bord du hapteur.

Cadre buccal (fig. 3).

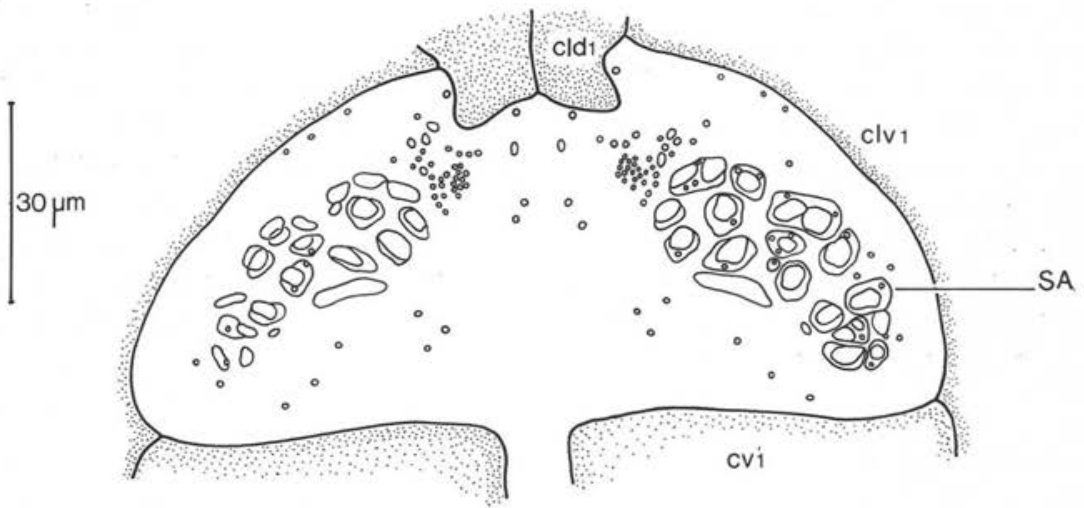

Fig. 3. Benedenia monticelli (Parona et Perugia, 1895). Dessin à la chambre claire du cadre buccal de l'oncomiracidium montrant l'aspect du « Système argyrophile » (SA).

Nomenclature des cellules ciliées: voir texte.

Le cadre buccal est ici parfaitement délimité par les cellules ciliées céphaliques qui l'entourent sauf sur un tout petit espace médio-ventral.

Les éléments argyrophiles y sont nombreux et polymorphes.

Une trentaine de sensilles de taille normale est disposée symétriquement dans toute cette zone. Dans la région antérieure certaines sont de taille très supérieure (4 à $6 \mu \mathrm{m}$ ) alors que d'autres au contraire, plus petites, sont regroupées en un amas : ces formations représentent des sensilles particulières ou des orifices glandulaires.

Certains éléments argyrophiles caractéristiques n'ont jamais été observés jusqu'à présent. De chaque côté, leur nombre varie de 15 à 18. Ces éléments sont arrondis ou allongés et de taille très variable (quelques $\mu \mathrm{m}$ à $15 \mu \mathrm{m}$ ). Chacun est limité par une ligne fermée argyrophile qui renferme une ou deux sous-unités sub-circulaires, de même aspect. Entre le cercle externe et les sous-unités il peut y avoir 1 à 3 sensilles. L'ensemble de ces éléments caractéristiques constitue ce que nous appelons le «sys- 
tème argyrophile buccal » qui jusqu'à présent n'a été mis en évidence que chez les Capsalidae.

Hapteur.

C'est la région du corps où les sensilles sont le plus difficile à observer; leur imprégnation étant semble-t-il plus inconstante. Il y a :

- 3 à 4 sensilles sur le bord du hapteur en avant des cellules ciliées haptoriales $(\mathrm{SH})$.

- 3 à 4 sensilles sur le bord du hapteur dans la région tout à fait postérieure (SH).

\section{II. - Entobdella soleae}

Nous donnons ici les premiers résultats sur cet oncomiracidium. Ils sont encore incomplets, car nous n'avons jamais eu l'imprégnation totale d'un seul spécimen. Les larves de cette espèce s'avèrent très difficiles à colorer à l'AgNO $\mathrm{N}_{3}$; cependant, en regroupant les observations faites sur plusieurs larves nous pouvons conclure:

1. - La couverture ciliée de l'oncomiracidium d'E. soleae est identique à celle de B. monticelli. En particulier les groupes céphaliques et pleuraux ont la même organisation et comportent le même nombre de cellules. Le groupe haptorial recouvre de la même manière l'extrémité dorsale du hapteur mais le nombre de cellules de cette zone reste à préciser.

2. - Le plan général de la chétotaxie correspond à celui décrit chez B. monticelli $:$ on y retrouve les mêmes groupes. Quelques différences existent: il y a une paire de sensilles supplémentaire plus postérieure, dorsalement (6 SD au lieu de $5 \mathrm{SD}$ chez B. monticelli). Sur quelques individus nous avons observé une paire de sensilles supplémentaire dans ce même groupe mais antérieurement (soit $7 \mathrm{SD}$ ).

$\mathrm{Au}$ niveau du cadre buccal le système argyrophile existe; cependant chacun des éléments semble contenir plus de sous-unités (jusqu'à 9 ou 10 au lieu de 3 chez B. monticelli).

Les groupes SDL, SDE, SDP et SV sont semblables à ceux de B. monticelli. La disposition des sensilles des groupes latéraux reste à préciser.

\section{III. - Trochopus pini}

Nous formulons pour cette espèce les mêmes remarques préliminaires que pour E. soleae mais les premiers résultats prouvent que chez l'oncomiracidium de $T$. pini :

1. - La couverture ciliée est identique à celle de B. monticelli et de E. soleae. Là aussi, le nombre des cellules du groupe haptorial reste cependant à préciser. 
2. - Le plan général de la chétotaxie est du même type que celui des espèces précédentes. Une sensille semble manquer dans le groupe dorsal (4 SD au lieu de 5 chez $B$. monticelli et de 6 à $7 \mathrm{chez}$ E. soleae). Les autres groupes se retrouvent ainsi que le système argyrophile buccal dont l'aspect est très proche de celui de $B$. monticelli.

\section{IV. - Discussion et conclusion}

L'étude détaillée de l'oncomiracidium de Benedenia monticelli et les premières observations sur les larves d'Entobdella soleae et Trochopus pini prouvent qu'il existe un plan commun de la chétotaxie et des cellules ciliées chez ces trois Capsalidae.

En comparant cette organisation à celle d'autres larves déjà décrites (Lambert, 1976, $1977 a$ et $b$ et 1978), nous remarquons qu'il existe des affinités avec «le type Dactylogyroïdea 》 (qui comprend les Dactylogyridae, Ancyrocephalidae et Diplectanidae décrits mais aussi Tetraonchus monenteron: Tetraonchidae). Mais il y a des différences dont quelques-unes paraissent importantes:

\section{1. - Cellules ciliées :}

Les cellules ciliées s'organisent de la même manière en 4 zones (une céphalique, 2 pleurales et une haptoriale). En particulier, les zones céphalique et pleurales comportent le même nombre de cellules (respectivement 27 et 2 fois 10). Chez les Capsaloïdea décrits, ces cellules sont toutes de grande taille et jointives alors que chez le type Dactylogyroïdea les cellules ciliées sont, dans chaque groupe, petites et plus séparées. Compte tenu de la taille des oncomiracidiums, il en résulte une augmentation de la surface corporelle ciliée larvaire chez les Capsalidae.

\section{2. - Chétotaxie :}

Dans les 2 plans d'organisation, il y a des groupes en commun (SD, SDE, SLA). Dans le type Capsaloïdea, on ne retrouve pas le grand nombre de sensilles médiodorsales qui caractérisent les Dactylogyroïdea (7 à 9 SD contre 4 à 6 chez les Capsalidae.

Il existe chez $B$. monticelli, E. soleae, $T$. pini un groupe de sensilles supplémentaire (SDL) qui n'existe chez aucun Dactylogyroïdea et inversement, nous n'avons pas retrouvé chez les Capsalidae les sensilles dorsales oculaires (SDO) des Dactylogyroïdea. Par contre, le groupe de sensilles supplémentaire (SDL) des Capsalidae se retrouve dans le type Polyopisthocotylea.

Le système argyrophile buccal est très caractéristique des Capsalidae et nous ne l'avons jamais observé chez d'autres Monogènes. Ce système argyrophile représenterait peut-être l'ébauche des ventouses ou des amas glandulaires latéraux qui existent de part et d'autre de l'extrémité antérieure des adultes. Ces organes sont essentiellement glandulaires chez $E$. soleae et musculaires, avec des glandes, chez 
B. monticelli et $T$. pini. Ceci expliquerait les différences observées chez la larve d'E soleae et celles de B. monticelli et $T$. pini.

A ce stade de l'analyse, il apparaît que le «type Capsalidae» est original; il présente certes quelques affinités avec le type Monopisthocotylea. Mais les différences avec les Dactylogyroïdea étudiés sont aussi importantes qu'avec les Polyopisthocotylea connus.

Nous conclurons en posant les questions suivantes :

- Le type Capsaloïdea correspond-il à un phylum original qui regrouperait des parasites cutanés ou secondairement branchiaux?

- Le type Capsaloïdea représente-t-il un schéma chétotaxique ontogénétiquement plus avancé comme cela est déjà le cas pour la structure du hapteur et le système excréteur larvaire?

Pour répondre à ces questions, nous nous proposons d'analyser l'évolution de la chétotaxie chez les post-larves des Monogènes, immédiatement après leur fixation sur l'hôte, et son évolution ultérieure.

\section{Bibliographie}

Lambert A. (1976): Mise en évidence et importance de la chétotaxie larvaire chez les Monogènes Monopisthocotylea. C.R. Acad. Sci. 282, sér. D, 1109-1112.

Lambert A. (1977 a): L'oncomiracidium d'Ancyrocephalus paraaoxus Creplin, 1839 (Monogenea, Monopisthocotylea) parasite de Sander lucioperca (Téléostéen, Percidae). Ann. Parasitol. Hum. Comp., 52, 493-505.

Lambert A. $(1977 b)$ : Recherches sur les affinités phylétiques des Polystomatidae. C.R. Acad. Sci. sér. $D, 1243-1246$.

Lambert A. (1978): Précisions sur l'oncomiracidium de Tetraonchus monenteron (Wagener, 1857) (Monogenea, Tetraonchidae) parasite d'Esox lucius L. (Téléostéen). Ann. Parasitol. Hum. Comp., $53,117-121$. 